\title{
Erratum to: Tonic GABAA Receptors as Potential Target for the Treatment of Temporal Lobe Epilepsy
}

\author{
S. Schipper ${ }^{1,2}$ - M. W. Aalbers ${ }^{2}$ - K. Rijkers ${ }^{2,3}$ - A. Swijsen ${ }^{4}$. J. M. Rigo ${ }^{4}$. \\ G. Hoogland ${ }^{2,5}$ - J. S. H. Vles ${ }^{1,2}$
}

Published online: 19 October 2015

(C) Springer Science+Business Media New York 2015

\section{Erratum to: Mol Neurobiol}

DOI: 10.1007/s12035-015-9423-8

The original paper of this article unfortunately contains error in Table 2. Some of the data were misplaced during the publication process. With this, the correct Table 2 is hereby presented.

S. Schipper

S.Schipper@maastrichtuniversity.nl

1 Department of Neurology, Maastricht University Medical Center, Maastricht, The Netherlands

2 Faculty of Health Medicine and Life Sciences, School of Mental, Health and Neuroscience, Maastricht University Medical Center, Maastricht, The Netherlands

3 Department of Neurosurgery and Orthopedic Surgery, Atrium Hospital Heerlen, Heerlen, The Netherlands

4 BIOMED Research Institute, Hasselt University/Transnational, University Limburg, Martelarenlaan 42, 3500 Hasselt, Belgium

5 Department of Neurosurgery, Maastricht University Medical Center, Maastricht, The Netherlands 
Table 2 Summary of the evidence concerning the role of subunit expression in temporal lobe epilepsy

\begin{tabular}{|c|c|c|c|c|c|c|c|c|}
\hline Author & Year & Model & Species & Technique & Cell type & Decrease & Increase & Additional findings \\
\hline Bouilleret & 2000 & $\begin{array}{l}\text { SE } \\
\text { KA i.c. }\end{array}$ & Mouse & IHC & $\begin{array}{l}\text { DG } \\
(\mathrm{GC} / \mathrm{ML}) \\
\mathrm{CA} 1 / 3\end{array}$ & $\alpha 5, \gamma 2$ & $\begin{array}{l}\gamma 2, \alpha 5 \\
\alpha 1\end{array}$ & $\begin{array}{l}\text { Loss of GAT-1 in CA1 } \\
\text { and DG, not in CA3 }\end{array}$ \\
\hline $\begin{array}{l}\text { Brooks- } \\
\text { kayal }\end{array}$ & 1998 & $\begin{array}{l}\text { SE } \\
\text { Pilocarpine }\end{array}$ & Rat & $\begin{array}{l}\text { Whole cell patch clamp, } \\
\text { Single-cell mRNA } \\
\text { amplification }\end{array}$ & DG & $\alpha 1(\mathrm{E})$ & $\alpha 4, \delta$ & $\begin{array}{l}\text { Altered sensitivity to } \\
\text { zolpidem and zinc } \\
\text { Increased } \\
\text { expression }\end{array}$ \\
\hline Drexel & 2013 & $\begin{array}{l}\text { SE } \\
\text { KA }\end{array}$ & Rat & In situ hybridization & $\begin{array}{l}\text { DG } \\
\text { CA1 } \\
\text { CA3 }\end{array}$ & $\begin{array}{l}\alpha 5, \delta \\
\gamma 2(\mathrm{E}), \delta \\
\alpha 5, \gamma 2(\mathrm{E})\end{array}$ & $\begin{array}{l}\alpha 4, \alpha 1 \\
\alpha 1\end{array}$ & \\
\hline Fritschy & 1999 & $\begin{array}{l}\text { SE } \\
\text { Pilocarpine }\end{array}$ & Rat & $\mathrm{IHC}$ & $\begin{array}{l}\text { DG } \\
(\mathrm{GC} / \mathrm{ML}) \\
\text { CA3 }\end{array}$ & $\begin{array}{l}\alpha 1 \\
\alpha 5\end{array}$ & $\begin{array}{l}\alpha 3, \alpha 5 \\
\gamma 2\end{array}$ & \\
\hline Goodkin & 2008 & $\begin{array}{l}\text { SE } \\
\text { Continuous } \\
\text { hippocampal } \\
\text { stimulation }\end{array}$ & Rat & $\begin{array}{l}\text { Whole cell patch clamp } \\
\text { (DG) }\end{array}$ & & & & $\begin{array}{l}\text { Maintenance of tonic } \\
\text { GABA currents } \\
\text { No reduction of } \delta \text { - } \\
\text { subunit expression }\end{array}$ \\
\hline Houser & 2003 & $\begin{array}{l}\text { SE } \\
\text { Pilocarpine }\end{array}$ & Rat & $\begin{array}{l}\text { IHC } \\
\text { In situ hybridization }\end{array}$ & $\mathrm{CA} 1 / 2$ & $\alpha 5$ & & \\
\hline Kamphuis & 1995 & $\begin{array}{l}\text { Amygdala } \\
\text { kindling }\end{array}$ & Rat & In situ hybridization & DG (GC) & $\gamma 2(\mathrm{~L})$ & $\begin{array}{l}\alpha 1 / 2 / 4 \\
(\mathrm{E}), \gamma 2 \\
(\mathrm{E})\end{array}$ & \\
\hline Lee & 2013 & $\begin{array}{l}\text { SE } \\
\text { pilocarpine }\end{array}$ & Rat & $\begin{array}{l}\text { Whole cell patch (DG) 2-3 } \\
\text { weeks after status } \\
\text { epilepticus }\end{array}$ & & & & $\begin{array}{l}\text { Increase tonic inhibition } \\
\text { in GC in DG }\end{array}$ \\
\hline Loup & 2000 & Human & $\begin{array}{l}\text { Human, } \\
\text { hippocampal } \\
\text { sclerosis }\end{array}$ & IHC & $\begin{array}{l}\text { DG } \\
\text { GC } \\
\text { ML }\end{array}$ & & $\begin{array}{l}\alpha 1, \gamma 2 \\
\alpha 2\end{array}$ & \\
\hline Naylor & 2005 & SE Pilocarpine & Rat & $\begin{array}{l}\text { Whole cell patch clamp } \\
\text { (DG) }\end{array}$ & & & & $\begin{array}{l}\text { Increase in tonic } \\
\mathrm{GABA}_{\mathrm{A}} \mathrm{R} \text { mediated } \\
\text { currents one hour after } \\
\mathrm{SE}\end{array}$ \\
\hline Nishimura & 2005 & $\begin{array}{l}\text { SE } \\
\text { Hippocampal } \\
\text { kindling } \\
\text { Self-sustained } \\
\text { limbic status } \\
\text { epilepticus }\end{array}$ & Rat & In situ hybridization & $\begin{array}{l}\text { DG (GC) } \\
\text { CA1 } \\
\text { CA3 }\end{array}$ & $\begin{array}{l}\alpha 5(\mathrm{E} / \mathrm{L}), \\
\delta(\mathrm{E} / \mathrm{L}) \\
\alpha 5(\mathrm{E} / \mathrm{L}) \\
\alpha 5(\mathrm{E} / \mathrm{L}), \\
\gamma 2(\mathrm{E})\end{array}$ & $\gamma 2(\mathrm{E})$ & \\
\hline
\end{tabular}

$D G$ hippocampal dentate gyrus, $E$ early, $G C$ granular cell, i.c. intracerebral, $I H C$ immunohistochemistry, $I N$ interneuron, $K A$ kainic acid, $M L$ molecular layer 


\begin{tabular}{|c|c|c|c|c|c|c|c|c|}
\hline Peng & 2004 & $\begin{array}{l}\text { SE } \\
\text { Pilocarpine }\end{array}$ & Mouse & $\mathrm{IHC}$ & $\begin{array}{l}\text { DG (ML) } \\
\text { DG (IN) }\end{array}$ & $\delta$ & $\delta$ & \\
\hline Rajasekeran & 2010 & $\begin{array}{l}\text { SE } \\
\text { Continuous } \\
\text { hippocampal } \\
\text { stimulation }\end{array}$ & Rat & $\begin{array}{l}\text { Patch-clamp } \\
\text { Western blot }\end{array}$ & DG & $\delta$ & $\alpha 4$ & $\begin{array}{l}\text { Tonic currents are } \\
\text { maintained in DG cells } \\
\text { post-SE by } \alpha 4 \gamma 2 \\
\text { receptors } \\
\text { Reduced neurosteroid } \\
\text { (allopregnanolone, } \\
\text { L655708) sensitivity in } \\
\text { epileptic DGC } \\
\text { Retention of } \delta \text {-subunit in } \\
\text { ER }\end{array}$ \\
\hline Scimemi & 2005 & $\begin{array}{l}\text { SE } \\
\text { Pilocarpine/KA }\end{array}$ & Rat & $\begin{array}{l}\text { Whole cell patch clamp } \\
\text { CA1 } \\
\text { IHC }\end{array}$ & $\mathrm{CA} 1 / 3$ & $\alpha 5$ & & $\begin{array}{l}\text { Maintenance of tonic } \\
\text { GABA currents }\end{array}$ \\
\hline Schwarzer & 1997 & $\begin{array}{l}\text { SE } \\
\text { KA }\end{array}$ & Rat & $\mathrm{IHC}$ & DG (ML) & $\alpha 2, \delta(\mathrm{E})$ & $\begin{array}{l}\alpha 1 / 2 / 4 / 5 \\
\delta, \gamma 2(\mathrm{~L})\end{array}$ & \\
\hline Sun & 2013 & $\begin{array}{l}\text { i.c. } \quad \mathrm{CTZ} \\
\text { injection }\end{array}$ & $\begin{array}{l}\text { Cell culture } \\
\text { Rat }\end{array}$ & $\begin{array}{l}\text { Whole cell patch clamp in } \\
\text { cultured hippocampal } \\
\text { neurons overexpressing } \\
\alpha 5 \beta 3 \gamma 2 \text { and } \alpha 6 \beta 3 \delta \\
\text { Field potentials in vivo }\end{array}$ & & & & $\begin{array}{l}\text { Overexpression } \alpha 5 \beta 3 \gamma 2 \\
\text { and } \alpha 6 \beta 3 \delta \text { resulted in } \\
\text { enhanced tonic } \\
\text { inhibition and reduced } \\
\text { epileptiform activity in } \\
\text { vitro } \\
\text { THIP }(5 \mu \mathrm{M}) \text { suppressed } \\
\text { epileptiform r burst } \\
\text { activity and behavioral } \\
\text { seizures }\end{array}$ \\
\hline Tsunashima & 1997 & $\begin{array}{l}\text { SE } \\
\text { KA }\end{array}$ & Rat & In situ hybridization & $\begin{array}{l}\text { DG (GC) } \\
\text { CA1 } \\
\text { CA3 }\end{array}$ & $\begin{array}{l}\alpha 5, \gamma 2(\mathrm{E}) \\
\delta(\mathrm{L}) \\
\alpha 5, \gamma 2(\mathrm{~L}) \\
\alpha 5, \gamma 2\end{array}$ & & \\
\hline Zhan & 2009 & SE Pilocarpine & $\begin{array}{l}\text { Rat } \\
\text { Mouse }\end{array}$ & $\begin{array}{l}\text { Whole cell patch clamp } \\
\text { (DG) }\end{array}$ & & & & $\begin{array}{l}\text { Increase in tonic } \\
\text { signaling } \\
\text { Tonic currents are } \\
\text { maintained by } \alpha 4 \beta \times \delta \\
\text { and } \alpha 5 \beta \times \gamma\end{array}$ \\
\hline Zhang & 2007 & SE Pilocarpine & Mouse & $\begin{array}{l}\text { Immunogold- } \\
\text { electronmicroscopy } \\
\text { Whole cell patch clamp }\end{array}$ & DG (GC) & $\delta$ & & $\begin{array}{l}\text { Shift of } \gamma 2 \text { towards } \\
\text { perisynaptic location } \\
\text { Magnitude of tonic } \\
\text { GABA currents } \\
\text { maintained }\end{array}$ \\
\hline
\end{tabular}

Table 2 (continued) 